\title{
GSTM1 and GSTP1 and respiratory health in asthmatic children exposed to ozone
}

\author{
I. Romieu*, M. Ramirez-Aguilar*, J.J. Sienra-Monge\# ${ }^{\#}$ H. Moreno-Macías*, B.E. del \\ Rio-Navarro\#, G. David ", J. Marzec`, M. Hernández-Avila* and S. London ${ }^{\star}$
}

ABSTRACT: Acute exposure to ozone has been related to a wide spectrum of health effects in susceptible individuals. Genetic factors may influence interindividual variation in ozone response. The current authors investigated the relationships between common polymorphisms in two genes involved in response to oxidative stress, i.e. glutathione S-transferases M1 (GSTM1) and P1 (GSTP1), and both respiratory symptoms and lung function in response to ozone among childhood asthmatics.

A total of 151 asthmatic children, who were participants in a randomised controlled trial of antioxidant vitamin supplementation in Mexico City, were studied. Children were genotyped using PCR methods and followed from October 1998-April 2000.

Increases in reported breathing difficulty were associated with ozone exposure in children with GSTM1 null (8\%, 95\% confidence interval (CI) 1-15\%, per 20-ppb increase in 1-h maximum daily average over 7 days) or GSTP1 Valine/Valine (Val/Val) genotypes (14\%, 95\% Cl 5-25\%). In children with both GSTM1 null and GSTP1 Val/Val genotypes, the increase in breathing difficulty associated with a 20-ppb increase in ozone exposure was even greater $(21 \%, 95 \% \mathrm{Cl} 5-39 \%)$. GSTP1 genotypes were not significantly associated with ozone-related lung function changes.

In conclusion, asthmatic children with glutathione S-transferase M1 null and glutathione S-transferase P1 Valine/Valine genotypes appear more susceptible to developing respiratory symptoms related to ozone exposure.

KEYWORDS: Asthmatic children, glutathione S-transferase M1, glutathione S-transferase P1, lung function, ozone, symptoms

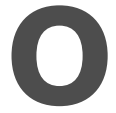
zone is a powerful oxidant. Antioxidants in the intracellular and extracellular spaces of the lung react with ozone and other oxidants to prevent conversion into more toxic species [1]. Despite these antioxidant defences, ozone reacts with lipids on cell membranes to produce secondary oxidation products [1]. Glutathione S-transferases (GSTs) conjugate such endogenous byproducts of oxidative stress with glutathione, enabling their rapid elimination and thus defending tissues against oxidant damage [2].

Common polymorphisms exist in genes coding for various GSTs including glutathione $S$ transferases M1 (GSTM1) and P1 (GSTP1). A common deletion of GSTM1 results in lack of the enzyme [3]. Homozygous deletion of the GSTM1 gene, also referred to as the null genotype, has been associated with: an increase of asthma and wheezing among children exposed to environmental tobacco smoke in utero [4]; a decrease in lung function growth in children [5]; and a rapid decline in lung function in smokers [6]. In addition, polymorphic GSTM1 has been shown to act as a modifier of the lung response to ozone [7]. GSTP1 is highly expressed in the lung and is also involved in response to oxidative stress [8]. A common polymorphism results in a substitution of valine (Val) for isoleucine (Ile) at codon 105, which forms part of the active site for binding of hydrophobic electrophiles, and affects substrate-specific catalytic activity [9]. Homozygotes for the Val allele have been shown to be at reduced risk of asthma in some studies [10, 11], including asthma induced by toluene di-isocyanate [10], but this finding is not consistent with other studies [12]. In contrast, the GSTP1 codon 105 Val allele has been reported to be associated with a significantly increased risk of cancer [13, 14].

\section{AFFILIATIONS}

*Instituto Nacional de Salud Publica, Cuernavaca, and

"Hospital Infantil "Federico Gómez", Mexico City, Mexico.

"Division of Intramural Research, National Institute of Environmental Health Sciences, National Institutes of Health, Dept of Health and Human Services, Research Triangle Park, NC USA.

CORRESPONDENCE

I. Romieu

Instituto Nacional de Salud Publica 655 Avenida Universidad

Col. Santa Maria Ahuacatitlán

62508 Cuernavaca

Morelos

México

Fax: 527773111148

E-mail: iromieu@correo.insp.mx

Received:

October 312005

Accepted after revision:

July 052006

\section{SUPPORT STATEMENT}

This study was supported by the Mexican Sciences and Technology Council (26206-M), the National Center for Environmental Health from the Centers for Disease Control and Prevention (Atlanta, GA, USA) and the Division of Intramural Research, National Institute of Environmental Health (Research Triangle Park, NC, USA; Z01 ES49019). The Roche Laboratory (Mexico City, Mexico) provided the supplements and placebos used in the study. I. Romieu was partly supported by the GA ${ }^{2} L E N$ project (EU contract FOODCT-2004506378) and the Spanish Ministry of Education and Science, SAB 20040192 
Mexico City experiences severe air pollution with high levels of ozone that frequently exceed the Mexican standard [15]. The current authors recently reported that asthmatic children had significant lung function changes after being subjected to ozone exposure that were modulated by antioxidant vitamins (vitamin C and vitamin E) [16]. Similarly, it was also found that GSTM1 null children had a greater decrease in forced midexpiratory flow (FEF25-75\%), a measure of pulmonary function, in response to ozone [17]. Given the role of GSTs in antioxidant defence, the present study investigated the effect of polymorphisms in both GSTM1 and GSTP1 on respiratory symptoms, asthma severity and pulmonary function in relation to ozone exposure among asthmatic children residing in Mexico City.

\section{METHODS AND MATERIALS}

\section{Study population}

Children with a diagnosis of asthma were recruited through the allergy clinic of the Hospital Infantil "Federico Gómez" (Mexico City, Mexico). The diagnosis of asthma was based on clinical symptoms and response to treatment. Using the Global Initiative on Asthma classification scheme, severity of asthma was graded as mild (intermittent or persistent), moderate and severe (National Institute of Health) [18, 19].

The study methods have been described previously [16]. Briefly, 160 children were recruited and followed up over a 12-week period. Parents were informed about the study and signed a consent form allowing their child to participate. At baseline, parents were interviewed and provided information on sociodemographic, health and risk factors for asthma, and dietary intake (food frequency questionnaire) of their child. Children performed a spirometric test and provided blood samples. Children were randomised to receive a supplement (vitamin C $250 \mathrm{mg} \cdot \mathrm{day}^{-1}$ and vitamin E $50 \mathrm{mg} \cdot \mathrm{day}^{-1}$ ) or placebo in a double-blind manner. None of the health personnel at the clinic nor the patients knew the randomisation status. Both placebo and supplements were presented in similar pills (Roche Laboratory, Mexico City, Mexico). During the 12-week follow-up, parents reported their child's daily respiratory symptoms in a diary that was reviewed at the clinic each week. In addition, children performed two spirometric tests per week at the clinic. Atopy was determined using skin-prick tests to 13 aeroallergens and nine food allergens. Reaction to histamine was used as positive control and glycerine as negative control. Children were considered atopic if the diameter of the skin reaction to at least one allergen exceeded $4 \mathrm{~mm}$. The test was considered valid if the reaction to histamine was $\geqslant 6 \mathrm{~mm}$ according to the grading of skin prick test recommended by AAS and BELIN [20].

The institutional review boards of the Hospital Infantil de Mexico, the Instituto Nacional de Salud Pública and the National Institute of Environmental Health Sciences approved this study. Of the 160 children, two were lost to follow-up because their mothers found it too inconvenient to come to the clinic, and for seven children a GSTP1 genotype could not be determined. Therefore, the current study reports results on 151 asthmatic children for whom there are complete data. For 139 children, a mean of 84 days of follow-up was available (range 81-92 days); 12 children had only 61 days of follow-up.

\section{Exposure assessment}

Measurements of ambient ozone, nitrogen dioxide, sulphur dioxide, particulates with a mass median diameter of $<10 \mu \mathrm{m}$ (PM10) and daily climatic variables (relative humidity and minimum, maximum and average temperature) were obtained from the Mexican government's air monitoring stations. The closest monitoring station to each child's residence was assigned to the child. None of the monitoring stations was further than $5 \mathrm{~km}$ from a child's residence. Data for sulphur dioxide were not analysed because of the low level of this contaminant.

\section{Genotyping}

DNA was extracted from whole blood or buffy coat using a standard GentraPuregene protocol (Gentra Systems, Minneapolis, MN, USA). GSTM1 genotype was determined by a PCR method as previously described [21]. The method distinguishes subjects with homozygous deletion of GSTM1 (null genotype) from those with either one or two copies of the gene (positive genotype) [21]. The three genotypes for the Ile105Val GSTP1 polymorphism (Ile/Ile $=\mathrm{AA}$, Ile $/ \mathrm{Val}=\mathrm{GA}$ or $\mathrm{Val} / \mathrm{Val}=\mathrm{GG}$ ) were determined by a Taqman allelic discrimination method. The polymorphic site was amplified from $50 \mathrm{ng}$ of genomic DNA using $600 \mathrm{nM}$ of each primer (forward primer, 5'-CCTGGTGGACATGGTGAATG-3'; reverse primer, $5^{\prime}$-TGCTCACATAGTTGGTGTAGATGA-3'), $200 \mathrm{nM}$ of each fluorescent probe (wildtype probe, 5'-6FAM-TGCAAATACATCTCCCT-3'; variant probe, 5'-VIC-CTGCAAATACGTCTCC-3'), and $10 \mu \mathrm{L}$ of Taqman ${ }^{\prime}$ 2X PCR Master Mix (Applied Biosystems, Foster City, CA, USA). Standard PCR cycling conditions were used, with initial denaturation at $95^{\circ} \mathrm{C}$ for $10 \mathrm{~min}$, followed by 35 cycles of $92^{\circ} \mathrm{C}$ for $15 \mathrm{~s}$ and $60^{\circ} \mathrm{C}$ for 1 min. Allele-specific PCR products were detected on an ABI 7700 (Applied Biosystems) and clustered by genotype using Sequence Detection System ${ }^{\circledR}$ software (Applied Biosystems). Ambiguous samples were manually clustered and verified by sequencing. Samples with known genotype, as verified by DNA sequencing, were run with each plate.

\section{Statistical analysis}

The Pearson correlation was determined between levels of air contaminants and various climatic variables [22]. The effect of ozone exposure on the probability of having respiratory symptoms on a given day was analysed using generalised estimation equations (GEE) for binary longitudinal data [23]. Different averages of ozone, nitrogen dioxide and PM10 exposure (1-h maximum, 24-h average, 8-h moving average) were used initially, but the maximum of the 1-h maximum exposure for these contaminants was used in the final analyses because of the high correlation between the different airpollutant levels. Respiratory symptoms and use of bronchodilator were categorised as present or absent on each day of the follow-up. The following variables were evaluated for confounding: age; sex; minimum temperature; asthma severity; exposure to environmental tobacco smoke; and chronological time. Significant predictors of respiratory symptoms were retained at $\mathrm{p}<0.10$. Interaction between ozone exposure and supplement groups on the occurrence of respiratory symptoms were tested for. These interaction terms were not significant; therefore, an indicator variable was used in the regression 
model to control for supplementation group. Interaction between ozone and GST polymorphisms (GSTM1 and GSTP1) was also tested for because these interactions were significant for different metrics of ozone exposure; the presented results are stratified by polymorphisms. The final models to study the impact of ozone on respiratory symptoms included: previous day minimum temperature (henceforth referred to as "temperature"); exposure to environmental tobacco smoke; asthma severity; chronological time; and supplementation group. The current authors previously reported that the GSTM1 genotype modified the spirometric response to ozone in these asthmatic children and that antioxidant supplementation modulated this effect [17]. The current authors also conducted a similar analysis to determine if GSTP1 genotypes also influence the susceptibility of asthmatic children to the spirometric effects of air pollutants using GEE [23] adjusting for the following variables: age; height; use of bronchodilator; respiratory symptoms on the day of the test; and chronological time.

GSTP1 Ile/Ile and Ile/Val were combined as they presented the same response pattern with regard to ozone exposure. To evaluate the combined effects of the GSTM1 and GSTP1 genotypes, children with both high-risk genotypes (GSTM1 null and GSTP1 Val/Val genotypes) were compared to children with both lower-risk genotypes (GSTM1 positive and GSTP1 Ile/Ile and Ile/Val). Finally, the significance of interaction terms between genotypes, supplementation groups and ozone was tested.

\section{RESULTS}

The distribution of asthma severity among the 151 asthmatics was $25.8 \% \quad(n=39)$ mild intermittent, $21.2 \% \quad(n=32)$ mild

\begin{tabular}{|c|c|c|c|c|c|}
\hline TABLE 1 & $\begin{array}{l}\text { line char } \\
1 \text { asthm } \\
-2000\end{array}$ & $\begin{array}{l}\text { cteris } \\
\text { c chil }\end{array}$ & $\begin{array}{l}\text { s of th } \\
\text { en res }\end{array}$ & $\begin{array}{l}\text { study } \\
\text { ng in }\end{array}$ & $\begin{array}{l}\text { ulation } \\
\text { co City, }\end{array}$ \\
\hline \multirow[t]{2}{*}{ Characteristic } & \multicolumn{2}{|c|}{ GSTM1 } & \multicolumn{3}{|c|}{ GSTP1 } \\
\hline & Positive & Null & Ile/Ile & Ile/Val & Val/Val \\
\hline Subjects $n$ & 93 & 58 & 31 & 66 & 54 \\
\hline Total \% & 61.6 & 38.4 & 20.5 & 43.7 & 35.8 \\
\hline Male \% & 62.4 & 63.8 & 61.3 & 62.1 & 64.8 \\
\hline \multicolumn{6}{|l|}{ Mean age at } \\
\hline \multicolumn{6}{|c|}{ Asthma severity \% } \\
\hline $\begin{array}{l}\text { Mild } \\
\text { Moderate and }\end{array}$ & 53.8 & 36.2 & 22.6 & 54.6 & 51.9 \\
\hline severe & 46.2 & $63.8^{\#}$ & 77.4 & 45.4 & $48.1^{\#}$ \\
\hline \multicolumn{6}{|l|}{ Treatment \% } \\
\hline Placebo & 50.5 & 46.6 & 54.8 & 42.4 & 53.7 \\
\hline Supplement & 49.5 & 53.4 & 45.2 & 57.6 & 46.3 \\
\hline \multicolumn{6}{|c|}{ Smoking at home \% } \\
\hline Father & 49.4 & 53.1 & 64.0 & 49.1 & 45.5 \\
\hline Mother & 21.9 & 18.2 & 36.4 & 18.5 & 14.6 \\
\hline
\end{tabular}

persistent, $49.0 \%(n=74)$ moderate, and $4.0 \%(n=6)$ severe. The GSTM1 null genotype was present in $38.4 \%$ of children. For GSTP1, 20.5\% $(n=31)$ had the Ile/Ile genotype, $43.7 \%$ $(\mathrm{n}=66)$ were Ile/Val and $35.8 \%(\mathrm{n}=54)$ were Val/Val. The GSTM1 and GSTP1 genotypes were not associated $(\mathrm{p}=0.70)$. Only three participants were nonatopic as assessed by skinprick tests. Positive skin tests were observed most frequently for dermatophagoides, cat, and German cockroach (Blatella germanica).

Major baseline characteristics of the study population are shown according to GSTM1 and GSTP1 polymorphisms in table 1. Children with the GSTM1 null genotype were more likely to suffer from moderate-to-severe asthma $(p=0.036)$ than GSTM1-positive children. Children with GSTP1 Ile/Ile genotype were also more likely to have moderate-to-severe asthma $(p=0.009)$ than children with GSTP1 Ile/Val or Val/Val genotypes. During the follow-up, children with a higher frequency of respiratory symptoms related to ozone also experienced a larger decrement in lung function (forced expiratory volume in one second (FEV1) and FEF25-75\% $(p<0.001)$ and forced vital capacity (FVC; $p=0.032)$ for association between lung function and respiratory symptoms).

\section{Exposure data}

Table 2 presents the levels of the air pollutants during the study period (October 1, 1998-April 30, 2000). The Mexican standard was exceeded for ozone (110 ppb 1-h maximum) on $41 \%$ of the days and for PM10 $\left(150 \mu \mathrm{g} \cdot \mathrm{m}^{-3} 24-\mathrm{h}\right.$ average $)$ on only $1.5 \%$ of the days. Ozone, PM10 and nitrogen dioxide were highly correlated. Correlations were $\mathrm{r}=0.57$ between ozone and nitrogen dioxide, $\mathrm{r}=0.45$ between ozone and PM10 and $r=0.57$ between nitrogen dioxide and PM10 $(p<0.0001$ for all coefficients).

\begin{tabular}{|c|c|c|c|}
\hline \multirow[t]{2}{*}{ TABLE 2} & \multicolumn{3}{|c|}{$\begin{array}{l}\text { Air pollution levels from the Mexico City } \\
\text { monitoring network during the study, 1998-2000 }\end{array}$} \\
\hline & Mean \pm SD & Min & $\operatorname{Max}$ \\
\hline \multicolumn{4}{|l|}{$\mathrm{O}_{3} \mathrm{ppb}$} \\
\hline 24-h average & $32 \pm 12$ & 7 & 80 \\
\hline 8-h moving average (max) & $69 \pm 31$ & 9.5 & 184 \\
\hline 1-h max & $102 \pm 47$ & 12 & 309 \\
\hline \multicolumn{4}{|l|}{$\mathrm{PM}_{10} \mu \mathrm{g} \cdot \mathrm{m}^{-3}$} \\
\hline 24-h average & $56.68 \pm 27.36$ & 9.92 & 249.06 \\
\hline 8-h moving average (max) & $81.08 \pm 39.22$ & 11.25 & 447.25 \\
\hline 1-h max & $137.42 \pm 78.67$ & 17 & 594 \\
\hline \multicolumn{4}{|l|}{$\mathrm{NO}_{2} \mathrm{ppb}$} \\
\hline 24-h average & $30 \pm 15$ & 5 & 110 \\
\hline 1-h max & $66 \pm 39$ & 8 & 298 \\
\hline Temp minimum ${ }^{\circ} \mathbf{C}$ & $10.06 \pm 3.38$ & 0.6 & 18.8 \\
\hline Max humidity \% & $53.58 \pm 17.02$ & 0.1 & 100 \\
\hline Min humidity $\%$ & $18.32 \pm 13.97$ & 0.1 & 73 \\
\hline
\end{tabular}




\begin{tabular}{|c|c|c|c|c|}
\hline & GSTM1 positive & GSTM1 null & GSTP1 Ile/lle Ile/Val & GSTP1 Val/Val \\
\hline Subjects $n$ & 93 & 58 & 97 & 54 \\
\hline \multicolumn{5}{|l|}{ Cough } \\
\hline Ozone 1-day lag & $0.99(0.97-1.01)$ & $1.01(0.99-1.04)$ & $0.99(0.97-1.01)$ & $1.01(0.98-1.04)$ \\
\hline Ozone 6-day average & $1.00(0.97-1.04)$ & $1.04(0.99-1.09)$ & $1.00(0.96-1.03)$ & $1.05(1.01-1.11)^{\#}$ \\
\hline \multicolumn{5}{|l|}{ Difficulty breathing } \\
\hline Ozone 1-day lag & $1.01(0.97-1.04)$ & $1.04(1.00-1.08)$ & $1.01(0.97-1.04)$ & $1.05(1.00-1.11)$ \\
\hline Ozone 2-day average & $1.02(0.98-1.07)$ & $1.05(1.00-1.10)$ & $1.01(0.97-1.04)$ & $1.09(1.02-1.16)^{\circ}$ \\
\hline Ozone 6-day average & $1.05(0.99-1.12)$ & $1.08(1.01-1.15)$ & $1.03(0.97-1.08)$ & $1.14(1.05-1.25)^{\natural}$ \\
\hline \multicolumn{5}{|l|}{ Bronchodilator use } \\
\hline
\end{tabular}

\section{Respiratory symptoms}

Table 3 presents the effect of ambient levels of ozone on respiratory symptoms according to genetic polymorphisms of GSTM1 and GSTP1. After adjusting for asthma severity, temperature, environmental tobacco smoke, chronological time and supplementation group, children with the GSTM1 null polymorphism were more likely to report difficulty breathing than GSTM1-positive children. Children with the GSTP1 Val/ Val genotype had a greater increase in breathing difficulty and bronchodilator use compared with children with the GSTP1 Ile/Ile and Ile/Val genotypes. This pattern was consistent for ozone exposure over various numbers of days of lag time. Among children with the GSTM1 null genotype, for an increase of $20 \mathrm{ppb}$ in the 1-h maximum ozone level over 6 days, the risk of breathing-difficulty episodes increased by $8 \%$ (95\% confidence interval (CI) 1-15\%; OR 1.08 95\% CI 1.011.15). In contrast, no significant increase in respiratory symptoms in relation to ozone was observed in children with a GSTM1-positive genotype. Among children with the GSTP1 Val/Val genotype, for an increase of $20 \mathrm{ppb}$ in the 1-h maximum ozone level over 6 days, breathing difficulty increased by $14 \%$ (95\% CI 5-25\%; OR 1.14 95\% CI 1.05-1.25), and use of bronchodilator increased by $5 \%$ (95\% CI 1-10\%; OR $1.0595 \%$ CI 1.01-1.11). Among children with GSTP1 Ile/Ile and Ile/Val genotypes, no significant increase in respiratory symptoms in relation to ozone was observed. The differences in the ozone effects between the GSTP1 Val/Val genotype and combined GSTP1 Ile/Ile and Ile/Val genotypes were statistically significant for both breathing difficulty and bronchodilator use $(\mathrm{p}<0.05$; table 3$)$. PM10 and nitrogen dioxide ambient levels were not related to breathing difficulty in any genotype groups; however, a significant increase in bronchodilator use was observed among children with the GSTP1 Val/Val genotype (see online Supplementary material).

A significant interaction was observed between combined genotypes of GSTM1 and GSTP1 and ozone ambient levels and the increase in the presence of breathing difficulty associated with these combinations was calculated. Children who had both GSTM1 null and GSTP1 Val/Val genotypes were more likely to suffer from breathing difficulty in relation to ozone exposure than children who were GSTM1 positive and had GSTP1 Ile/Ile and Ile/Val genotypes (table 4). An increase of $20 \mathrm{ppb}$ in 1-h maximum ozone average over 6 days was related to a $22 \%$ increase in the presence of breathing difficulty $(95 \%$ CI 7-40\%; OR 1.22 95\% CI 1.07-1.40). Among children who were GSTM1 positive and had GSTP1 Ile/Ile or Ile/Val genotypes, there was no significant increase in the risk of breathing difficulty with ozone exposure. The difference in the effect of ozone on the presence of breathing difficulty between children with GSTM1 null and GSTP1 Val/Val genotypes and children with GSTM1-positive and GSTP1 Ile/Ile or Ile/Val genotypes was statistically significant $(p<0.05)$. No significant differences were observed between these combinations of genotypes for cough or bronchodilator use.

It was not possible to thoroughly explore the effect of antioxidant supplementation on respiratory symptoms in relation to genotype because of the small number of events once data were stratified by supplement groups.

\section{Pulmonary function}

Ozone concentration on the day prior to the spirometric test was not significantly related to changes in lung function in 
TABLE 4 Effect of ozone (20 ppb) on the risk of reporting difficulty breathing on a given day according to combined GSTM1 and GSTP1 genotypes among the study population

GSTM1 null and GSTP1 Val/Val

\section{Subjects $n$}

Ozone

$\begin{array}{ll}\text { 1-day lag } & 1.08(1.00-1.17) \\ \text { 2-day average } & 1.12(1.02-1.23) \\ \text { 6-day average } & 1.22(1.07-1.40)\end{array}$
GSTM1 positive and GSTP1 Ile/lle and Ile/Val

Data are presented as odds ratio (95\% confidence interval). GSTM1: glutathione S-transferase M1; GSTP1: glutathione S-transferase P1; lle: isoleucine; Val: valine. Odds ratio and $95 \%$ confidence interval calculated using generalised estimation equations for logistic regression separately for each genotype cross-classification adjusting for previous day temperature and chronological time. ${ }^{\#}: p \leqslant 0.05 ; p$-value obtained comparing odds ratios within ozone exposure categories between combined genotype groups (GSTM1 null and GSTP1 ValNal versus GSTM1 positive and GSTP1 Ile/lle, Ile/Nal).

TABLE 5 Effect of ozone concentrations on the day prior to spirometric test on lung functions according to glutathione S-transferase P1 (GSTP1) polymorphisms among the study population

\begin{tabular}{|c|c|c|c|}
\hline Lung function & Group & Subjects $n$ & Coefficient $(95 \% \mathrm{Cl}) \mathrm{mL} 20 \mathrm{ppb} \mathrm{O}_{3}{ }^{-1}$ \\
\hline \multirow[t]{2}{*}{ FEF25-75\% } & GSTP1 Val/Nal & 54 & $-4.5(-16.1-7)$ \\
\hline & GSTP1 lle/lle lle/Nal & 97 & $-7.3(-16.3-1.8)$ \\
\hline FEV 1 & GSTP1 Ile/lle Ile/Val & 97 & $-4.3(-9-0.5)$ \\
\hline \multirow[t]{2}{*}{ FVC } & GSTP1 Val/Nal & 54 & $8.4(0.7-16.1)$ \\
\hline & GSTP1 Ile/lle lle/Nal & 97 & $-2.7(-8.6-3.2)$ \\
\hline
\end{tabular}

either GSTP1 Val/Val or GSTP1 Ile/Ile and Ile/Val. Similar results were observed over different lags (table 5).

\section{DISCUSSION}

The present results suggest that GSTM1 and GSTP1 genotypes modify the effect of ozone exposure on respiratory symptoms among asthmatic children. Children with GSTM1 null and/or GSTP1 Val/Val genotypes were more likely to experience respiratory symptoms in response to ozone. The difference was more apparent for the GSTP1 than GSTM1 polymorphisms: breathing difficulty and bronchodilator use differed significantly by GSTP1 genotype. In children having both GSTM1 null and GSTP1 Val/Val genotypes the risk of breathing difficulty was even larger. The current authors have previously reported that children with the GSTM1 null genotype had greater decreases in pulmonary function than GSTM1-positive children. In the current analysis, ozone concentration was not significantly related to lung function changes in either GSTP1 Val/Val or GSTP1 Ile/Ile and Ile/Val genotypes.

These results support emerging data about the role of GSTs in protection against oxidative stress [2]. GSTs catalyse the conjugation of glutathione to secondary oxidation products produced by exposure of lipids to environmental oxidants such as ozone [2]. In addition, GSTs can also influence oxidative stress by mechanisms other than conjugation with glutathione. For example, $\mathrm{CHO}$ et al. [24] recently found that GSTM1 may play an important role in modulating stressinduced signalling by repressing apoptosis signal-regulating kinase-1. The recent discovery that GSTs, including GSTM1, are induced in mitochrondria after exposure to lipid peroxidation products may be particularly relevant to the response to ozone [25]. GSTP1 is highly expressed in the human lung, while GSTM1 is expressed to a lesser extent [26].

In the current study, two highly functional polymorphisms were examined. The GSTM1 null genotype is a homozygous deletion of the GSTM1 gene resulting in loss of enzyme activity [3]. For GSTP1, the substitution of valine for isoleucine results in reduced enzyme activity, which is more pronounced when two copies of the valine allele are present [27]. The present authors would predict that the GSTM1 null subjects and those carrying the GSTP1 Val allele might be at an increased risk of oxidative stress because of lower levels of these two enzymes. The results of the current study are consistent with this prediction.

Very few studies have examined polymorphisms in xenobiotic metabolising genes as modifiers of the effect of pollutants on lung health. In utero exposure to maternal smoking has been 
related to asthma and wheezing occurrence in children with the GSTM1 null genotype [28]. The current authors recently reported that the GSTM1 null genotype was associated with an increased susceptibility to the effect of ozone on FEF25-75\%, a measure of lung function [17].

Thus, it has been observed that GSTP1 Val/Val homozygotes were more susceptible to the effect of ozone than children with the Ile/Ile and Ile/Val genotypes. Two previous studies, including one of toluene-induced occupational asthma, have suggested that subjects with the GSTP1 Val/Val genotype have reduced risk of asthma compared to Ile/Ile subjects [29, 30], and GILLILAND et al. [28] reported that GSTM1 null and GSTP1 Ile/Ile genotypes conferred increased susceptibility to diesel exhaust particles; none of the 19 subjects in this past study had the Val/Val genotype. In the Children's Health Study, the GSTM1 null genotype was associated with deficits in annual growth rates for FVC. Children with the GSTP1 Val/Val genotype had slower lung function growth (for FVC and FEV1) than children with one or more Ile 105 alleles. Larger deficits were observed in asthmatic children [5], suggesting that these children might be more susceptible to the impact of air pollutant on lung growth. In a study from Taiwan of 61 asthmatic children [31], the GSTP1 Ile/Ile genotype appeared to increase asthma risk in the presence of higher levels of air pollution exposure; however, only seven subjects in this study had the GSTP1 Val/Val genotype and therefore could not be studied separately. In contrast, atopic asthma has been related to GSTP1 Val/Val genotype in a Turkish population [12]. Subjects with GSTP1 Val/Val genotypes have also been shown to be more likely to develop lung cancer associated with exposure to tobacco [32] and environmental tobacco smoke [33]. In the current study, children with the GSTP1 Ile/Ile genotype were more likely to have moderate-to-severe asthma, although numbers in that category were small. However, the effect of ozone exposure was stronger in children with GSTP1 $\mathrm{Val} / \mathrm{Val}$ genotype on respiratory symptoms. The differences among studies may reflect the play of chance or different populations and ethnic groups. In the current study population, almost all children were atopic and the prevalence of Val/Val genotype was high (35.8\%). Significant association between ozone concentrations and changes in lung functions in relation to GSTP1 genotypes was not observed. This might be due to the limited sample size and hence the results of the current study should be interpreted with caution.

Several issues need to be considered in the interpretation of the results. The measure of ozone exposure was based on ambient monitoring stations located within $5 \mathrm{~km}$ of the children's homes which might have led to misclassification of exposure. However, a validation study was conducted using personal monitors and a significant association between personal exposure and ambient ozone levels was observed $(p<0.01)$. The assessment of respiratory symptoms is subjective, but the current authors believe that the information provided on respiratory symptoms is unlikely to be subject to reporting bias given that mothers did not know their child's genotype nor the levels of ozone on previous days. Therefore, misreporting should be random and would underestimate the associations.

A protective effect of antioxidant supplementation on respiratory symptoms related to ozone exposure among GSTM1 null children was observed in the current study, in a similar manner to that previously observed for lung function changes [17]. In contrast, a consistent protective effect of antioxidant supplementation on respiratory symptoms was not presently observed in relation to ozone in children with GSTP1 Val/Val genotypes.

As in any outbred population, population stratification is possible. Mexicans are predominantly a mix of Amerindian and Caucasian ethnicity [34]. However, the primary finding is that genotypes of GSTM1 and/or GSTP1 may modify the association between ozone exposure and pulmonary responses. For population stratification to have created this apparent effect modification by GST genotypes, one would have to postulate that a very strong correlate of the underlying population structure is the true effect modifier. This unknown susceptibility factor would also need to be nearly perfectly correlated with both the GSTM1 and GSTP1 genotypes to have created the apparent effect modification by each of these genes. This scenario seems highly unlikely.

In conclusion, the current data provide preliminary evidence that asthmatic children with glutathione $S$-transferase M1 null and glutathione S-transferase P1 valine homozygous genotypes may be more susceptible to the impact of ozone exposure on their lungs.

\section{ACKNOWLEDGEMENTS}

The authors would like to thank the Monitoring Network of Mexico City for providing data on air pollutants and H. Li for genotyping.

\section{REFERENCES}

1 Mudway IS, Kelly FJ. Ozone and the lung: a sensitive issue. Mol Aspects Med 2000; 21: 1-48.

2 Hayes JD, Flanagan JU, Jowsey IR. Glutathione transferases. Annu Rev Pharmacol Toxicol 2005; 45: 51-88.

3 Rebbeck TR. Molecular epidemiology of the human glutathione S-transferase genotype GSTM1 and GSTT1 in cancer susceptibiltity. Cancer Epidemiol Biomarkers Prev 1997; 6: 733-743.

4 Gilliland FD, Lin Y, Dubeau L, et al. Effects of glutatione S-transferase M1, maternal smoking during pregnancy and environmental tobacco smoke on asthma and wheezing in children. Am J Respir Crit Care Med 2002; 166: 457-463.

5 Gilliland FD, Guaderman J, Vora H, Rappaport E, Dubeau L. Effects of glutathione S-transferase M1, T1, and P1 on childhood lung function growth. Am J Respir Crit Care Med 2002; 166: 710-716.

6 He JQ, Ruan J, Connett JE, Anthonisen NR, Paré PD, Sandford AJ. Antioxidant gene polymorphisms and susceptibility to a rapid decline in lung function in smokers. Am J Respir Crit Care Med 2002; 166: 323-328.

7 Corradi M, Alinovi R, Goldoni M, et al. Biomarkers of oxidative stress after controlled human exposure to ozone. Toxicol Lett 2002; 134: 219-225.

8 Zimniak P, Nanduri B, Pikula S, et al. Naturally occurring human glutathione S-transferase GSTP1-1 isoforms with isoleucine and valine in position 104 differ in enzymic properties. Eur J Biochem 1994; 224: 893-899. 
9 Johansson AS, Stenberg G, Widersten M, Mannervik B. Structure-activity relationships and thermal stability of human glutathione transferase P1-1 governed by the H-site residue 105. J Mol Biol 1998; 278: 687-698.

10 Mapp CE, Fryer AA, De Marzo N, et al. Glutathione S-transferase GSTP1 is a susceptibility gene for occupational asthma induced by isocyanates. J Allergy Clin Immunol 2002; 109: 867-872.

11 Hemmingsen A, Fryer AA, Hepple M, Strange RC, Spiteri MA. Simultaneous identification of GSTP1 Ile105$>$ Val105 and Ala114->Val114 substitutions using an amplification refractory mutation system polymerase chain reaction assay: studies in patients with asthma. Respir Res 2001; 2: 255-260.

12 Tamer L, Calikoglu M, Ates NA, et al. Glutathione-Stransferase gene polymorphisms (GSTT1, GSTM1, GSTP1) as increased risk factors for asthma. Respirology 2004; 9: 493-498.

13 Harries LW, Stubbins MJ, Forman D, Howard GC, Wolf CR. Identification of genetic polymorphisms at the glutathione S-transferase Pi locus and association with susceptibility to bladder, testicular and prostate cancer. Carcinogenesis 1997; 18: 641-644.

14 Helzlsouer KJ, Selmin O, Huang HY, et al. Association between glutathione S-transferase M1, P1, and T1 genetic polymorphisms and development of breast cancer. J Natl Cancer Inst 1998; 90: 512-518.

15 O'Neill MS, Ramírez-Aguilar M, Meneses-Gonzalez F, et al. Ozone exposure among Mexico City outdoor workers. J Air Wasta Manag Assoc 2003; 53: 339-346.

16 Romieu I, Sienra-Monge JJ, Ramirez-Aguilar M, et al. Antioxidant supplementation and lung functions among children with asthma exposed to high levels of air pollutants. Am J Respir Crit Care Med 2002; 166: 703-709.

17 Romieu I, Sienra-Monge JJ, Ramirez-Aguilar M, et al. Genetic polymorphism of GSTM1 and antioxidant supplementation influence lung function in relation to ozone exposure in asthmatic children in Mexico City. Thorax 2004; 59: 8-10.

18 British Thoracic Society, Scottish Intercollegiate Guidelines Network. British Guideline on the Management of Asthma. Thorax 2003; 58: Suppl. 1, 1-94.

19 Pocket Guide for Asthma Management and Prevention: Global Initiative for Asthma. Publication NIH No. 963659B. Bethesda, National Institutes of Health, 1998.

20 Aas K, Belin L. Standardization of diagnostic work in allergy. Int Arch Allergy Appl Immunol 1973; 45: 57-60.

21 Bell DA, Taylor JA, Paulson DF, Robertson CN, Mohler JL, Lucier GW. Genetic risk and carcinogen exposure: a common inherited defect of the carcinogen-metabolism gene glutathione S-transferase M1 (GSTM1) that increases susceptibility to bladder cancer. J Natl Cancer Inst 1993; 85 1159-1164.

22 Zar J. Biostatistical analysis. 3rd Edn. Upper Saddle River, Prentice Hall, 1996.

23 Diggle PJ, Liang KY, Zeger SL. Analysis of Longitudinal Data. Oxford, Oxford University Press, 1994.

24 Cho SG, Lee YH, Park HS, et al. Glutathione S-transferase mu modulates the stress-activated signals by suppressing apoptosis signal-regulating kinase 1. J Biol Chem 2001; 276: 12749-12755.

25 Hayes JD, Strange RC. Glutathione S-transferase polymorphisms and their biological consequences. Pharmacology 2000; 61: 154-166.

26 Cantlay AM, Smith CA, Wallace WA, Yap PL, Lamb D, Harrison DJ. Heterogeneous expression and polymorphic genotype of glutathione S-transferases in human lung. Thorax 1994; 49: 1010-1014.

27 Watson MA, Stewart RK, Smith GB, Massey TE, Bell DA. Human glutathione S-transferase P1 polymorphisms: relationship to lung tissue enzyme activity and population frequency distribution. Carcinogenesis 1998; 19: 275-280.

28 Gilliland FD, Rappaport EB, Berhane K, et al. Effects of glutathione S-transferase P1, M1, and T1 on acute respiratory illness in school children. Am J Respir Crit Care Med 2002; 166: 346-351.

29 Fryer AA, Bianco A, Hepple M, Jones PW, Strange RC, Spiteri MA. Polymorphism at the glutathione S-transferase GSTP1 locus. A new marker for bronchial hyperresponsiveness and asthma. Am J Respir Crit Care Med 2000; 161: 1437-1442.

30 Gilliland FD, Li Y, Saxon A, Diaz-Sanchez D. Effect of glutathione-S-transferase M1 and P1 genotypes on xenobiotic enhancement of allergic responses: randomised, placebo-controlled crossover study. Lancet 2004; 363: 119-125.

31 Lee YL, Lin YC, Lee YC, Wang JY, Hsiue TR, Guo YL. Glutathione S-transferase P1 gene polymorphism and air pollution as interactive risk factors for childhood asthma. Clin Exp Allergy 2004; 34: 1707-1713.

32 Miller DP, Neuberg D, de Vivo I, et al. Smoking and the risk of lung cancer susceptibility with GSTP1 polymorphism. Epidemiology 2003; 14: 545-551.

33 Miller DP, De Vivo I, Neuberg D, Wain JC, Lynch TJ, Christiani DC. Association between self-reported environmental tobacco smoke exposure and lung cancer: modification by GSTP1 polymorphism. Int J Cancer 2003; 104: 758-763.

34 Choudhry S, Coyle NE, Tang $\mathrm{H}$, et al. Population stratification confounds genetic association studies among Latinos. Hum Genet 2006; 118: 652-664. 